\title{
Management of Post-Consumer Pharmaceutical Waste: An Overview
}

\author{
Imad Eldin Mohamed Nour ${ }^{1 *}$, Asim Ahmed Elnour ${ }^{2}$, TasneemJaafarMahjoub Salih ${ }^{3}$, \\ WadhaMohmaed Al Mulla ${ }^{4}$ and Akshaya Srikanth Bhagavathula ${ }^{5}$ \\ ${ }^{1}$ Ministry of Health, Directorate General of Pharmaceutical Affairs and Drug Control, Sultanate of Oman. \\ ${ }^{2}$ Faculty,Program Lead,Pharmacy Program, Fatima College of Health Sciences, Al Ain-UAE \\ ${ }^{3}$ Internship Pharmacist, Pharmalink-Medicina group, Abu Dhabi, UAE
}

${ }^{4}$ Pharmacist (Resident), Department, Sheikh Khalifa Medical City, Services Health Authority-SEHA, Abu Dhabi-UAE

${ }^{5}$ Assistant Professor of Clinical Pharmacy, University of Gondar-College of Medicine and Health Sciences, Gondar, Ethiopia

Received: November 02, 2017; Accepted: December 04, 2017; Published: December 08, 2017

*Corresponding author: ImadEldin Mohamed Nour, Ministry of Health, Directorate General of Pharmaceutical Affairs and Drug Control, Sultanate of Oman.Tel: +96899791465; E-mail: imdnor@hotmail.com; imad.nour@moh.gov.om

\begin{abstract}
Background: Post-consumer pharmaceutical waste (unused and expired pharmaceuticals) can have a remarkable hazard impact on our environment if not properly treated and disposed.

Aim of the review: In this review, we intend to explore the issue of pharmaceutical disposal, bylaws, safety, confidentiality and available means.

Methods: A search in the literature was conducted to gather and compare data about the pharmaceutical waste management in various regions in the globe, including; Europe, Australia, The United States, Canada, Saudi Arabia, United Arab Emirates and Oman.

Results: In USA the Drug Administration Agency-FDA published a disposal protocol on their website for Americans to follow if their community does not have a return program. The European Union has adopted a risk assessment policy for managing PhACs. In 1989, the Canadian Council of Ministers of the Environment-CCME deemed waste management a problem and developed a national packaging policy to reduce packaging waste sent to landfills. In UAE, industry experts said the wastage occurs most commonly with painkillers, paracetamol, anti-histamines, cough syrups and nasal sprays. Recently, the same issue has come to light in an article published in the Oman Daily Observer with the heading "Medicines worth millions go waste".

Conclusion: Health authorities need to develop disposal programs as part of pharmaceuticals use analysis in health strategies and community initiatives to promote vigilant culture for undesirable environmental consequences in the developing and underdeveloped countries. Pharmacists have to take proactive role in minimizing and ensuring safe disposal of pharmaceutical waste. Finally, the key to solve the issue of pharmaceutical waste disposal is collaborative effort and co-operation between all the parties in the health care hierarchy. Established and applied laws, along with community awareness will definitely make our aim easily achievable.
\end{abstract}

Key words: Pharmaceutical Waste; Pharmacist; Disposal of Pharmaceutical Waste; Post Consumer;

\section{Introduction}

Post-consumer pharmaceutical waste (unused and expired pharmaceuticals) can have a remarkable hazard impact on our environment if not properly treated and disposed. Numerous consumers dispose their unused pharmaceuticals in the trash or down the drain. However; not only consumers, even some healthcare facilities, hospitals and clinics may dispose their unused pharmaceuticals on the same manner. Since sewage treatment plants are not designed to treat all pharmacologically active compounds (PhAC's), these substances can pass through the treatment process and are then released into the environment. Over the past two decades several studies have detected PhAC's in ground water, surface water, soil and drinking water, [1, 2, 3].
The disposal of pharmaceutical waste is a sensitive, growing concern, since the type of waste it is dealing with is active pharmaceutical compounds that exert a pharmacological effect on living beings and the environment. The pharmaceutical waste includes expired, unused and contaminated pharmaceuticals, [4, 5] that cannot be reused. The fate of this type of waste is nothing other than a proper destruction or removal procedure that ensures the disposal of pharmaceutical waste without negatively impacting the environment. 
Scientific uncertainties exist regarding the risk of PhAC's in the environment and scientist have not yet quantified the human health risk of these compounds. However; concern about the health impacts associated with exposure to a mixture of pharmaceuticals in the environment, certainly exist. It is deemed that both scientists and policy makers take the precautionary measures to protect both the environment and human health. The gap in the third world underdeveloped and developing countries is broader, as there is neither proper treatment for post-consumer pharmaceutical waste, nor awareness or concern about its health hazard impact.

\section{Aim of the Review}

In this review, we intend to explore the issue of pharmaceutical disposal, bylaws, safety, confidentiality and available means. We discuss the current challenges and possible measures that can be adopted to control disposal of pharmaceuticals. The role of pharmacist's in disposal of pharmaceuticals was emphasized with special attention.

\section{Methods}

A search in the literature was conducted to gather and compare data about the pharmaceutical waste management in various regions in the globe, including; Europe, Australia, The United States, Canada, Saudi Arabia, United Arab Emirates and Oman. A total of 50 articles were reviewed of which 23 were relevant and identified from three online databases: PubMed, Scopus and Science Direct. The search strategy focused on obtaining articles with: "pharmaceutical waste", "Post-consumer pharmaceutical waste", "pharmaceutical waste management", "unwanted medications" and "drug disposal" as article headings. Then these keywords were matched with the countries and regions intended to be studied as headings or text words. Papers published in English language between 1998 and 2016 were considered.

\section{Results}

In USA the Drug Administration Agency-FDA published a disposal protocol on their website for Americans to follow if their community does not have a return program. The European Union has adopted a risk assessment policy for managing PhACs. In 1989, the Canadian Council of Ministers of the EnvironmentCCME deemed waste management a problem and developed a national packaging policy to reduce packaging waste sent to landfills. In UAE, industry experts said the wastage occurs most commonly with painkillers, paracetamol, anti-histamines, cough syrups and nasal sprays. Recently, the same issue has come to light in an article published in the Oman Daily Observer with the heading "Medicines worth millions go waste".

\section{The World Health Organization (WHO) status}

In 1999, the World Health Organization (WHO) adopted Guidelines for Safe Disposal of Unwanted Pharmaceuticals' in and after Emergencies, [6]. These guidelines provide advice on the implementation of safe disposal of unusable pharmaceuticals in emergencies and in countries in transition where official assistance and advice may not be available. They are not meant to supersede local, regional or national laws regarding disposal of pharmaceuticals, but to provide assistance where there is insufficient guidance or none at all.

During conflicts and natural disasters, large quantities of pharmaceuticals are often donated as part of humanitarian assistance. Undoubtedly, many of the pharmaceuticals save lives and alleviate suffering, but some donations given by well-meaning may cause some problems for the uninformed people. Pharmaceuticals may arrive past or near their expiry date, may be inappropriate for the needs, been unrecognizable because they are labeled in a foreign language or may have been sent in unwanted quantities. Donated pharmaceuticals with a long shelf life may be mismanaged, particularly in the confusion during and after armed conflict or a natural disaster. Staff and storage space may be lacking and the pharmaceutical management system in disarray. Such problems also occur when pharmaceutical donations form part of development assistance. Smaller quantities of pharmaceutical waste may accumulate in the absence of emergency situations, due to inadequacies in stock management and distribution, and to lack of a routine system of disposal. Safe disposal of these unwanted or expired pharmaceuticals often creates a major problem. The size of the pharmaceutical waste in global pharmaceuticals market is worth US $\$ 300$ billion a year, a figure that is expected to rise to US $\$ 400$ billion within three years, [7].

\section{The international scope}

Developed countries in Europe and Australia, United State of America-USA and Canada have policy tools and instruments in place for managing this environmental problem. In 2004, the European Union (EU) adopted Directive 2004/27/EC which stated that "Member States shall ensure that appropriate collection systems are in place for medicinal products that are unused or have expired" (Article 127b). This Directive also mandates that the outer packaging (or immediate packaging) of medicinal products shall include "specific precautions relating to the disposal of unused medicinal products or waste derived from medicinal products, where appropriate, as well as reference to any appropriate collection system in place" (Article 54j).

\section{The European trend}

The European Environment Agency (EEA) conducted a survey in 2008, consisting of 28 countries, to investigate the amount of waste collected through medications return programs, [8]. They found that pharmacies are the most common return site. Nations with the lowest collection rates were Croatia 0.19 g/capita annually) and Estonia (3.4 g/capita annually), while countries with the highest collection rates were Switzerland (237 g/capita annually) and France (231 g/capita annually). According to Vollmer, [9] "The collection rate depend on the amount of pharmaceuticals distributed, variance in patients' compliance to use the prescribed pharmaceuticals for therapy and lack of knowledge of established return schemes or the environmental effects of pharmaceuticals flushed into the drain", (Vollmer, 2010; p. 176). Even though return programs have been widely 
established throughout Europe, the EEA still believed that a substantial volume of unused pharmaceuticals are not collected.

The EU has also adopted a risk assessment policy for managing PhACs. Producers are required to conduct an environmental risk assessment when applying for marketing authorization for human medicinal products (Directive 2001/83/EC) and veterinary medicinal products (Directive 2001/82/EC). To gain authorization to place a medicinal product for human use in the market, the application must accompany an "[evaluation of the potential environmental risks posed by the medicinal product] This impact shall be assessed and, on a case-by-case basis, specific arrangements to limit it shall be envisaged" (Directive 2001/83/ EC, Article 8). The overall purpose of the ERA is to quantify the potential environmental risks of a drug.

\section{Australian experience}

Since 1999, Australia has operated a national return program for the community's unused/expired drugs called the Return Unwanted Medicines (RUM) project. This program is facilitated by the National Return and Disposal of Unwanted Medicines Limited Organization. Consumers are encouraged to return their waste to pharmacies (at no additional cost), while pharmaceutical wholesalers provide financial support through a nominal fee for the collection and transportation of waste to and from pharmacies, [10]. The RUM project is primarily funded by the Federal government; however, some drug manufacturers have provided sponsorship support. In 2009, the Federal Budget approved over $\$ 9$ million for the RUM project over four years. In 2000-2001, about 235,000 kg of unused drugs was collected, while later in 2011-2012, 576,000 kg was collected, [10].

A RUM survey conducted in 2005 by Brushin, [11] found that about $68.4 \%$ of drugs returned through RUM; were solid (tablets and lozenges), roughly $11.8 \%$ were liquid (drops and solutions); and $5 \%$ were semi-solid (creams and ointments). The most common drugs returned were for treating the cardiovascular system $(\sim 20 \%)$, nervous system $(\sim 20 \%)$ and alimentary tract and metabolism $(\sim 15 \%)$. The five most common reasons for returning drugs were due to: the drug's efficacy e.g. the drug expired (32.9\%); death of the consumer (27.1\%); a modification in treatment $(13.1 \%)$; the consumer's perception regarding the need for the drug $(9.2 \%)$; and the consumer experienced unwanted effects $(8.5 \%)$.

\section{The USA contribution}

The USA does not have a national medications return program. Take-back schemes take place across communities at the local government level. The Secure and Responsible Drug Disposal Act of 2010, which amended the Controlled Substances Act, allows the US Drug Enforcement Administration (DEA) "to develop permanent, ongoing, and responsible methods for disposal",[12]. Before the Secure and Responsible Drug Disposal Act was enacted, Federal law did not permit take-back programs to accept controlled substances unless they received special approval from the US DEA and arranged for full-time law enforcement officers to receive the controlled substances directly from the consumer
(Secure and Responsible Drug Disposal Act of 2010).Hence, many of the return initiatives would take place at Police Departments. The Food and Drug Administration Agency-FDA published a disposal protocol on their website for Americans to follow if their community does not have a return program, [13]. They advise consumers to mix the drugs with an unpalatable substance like kitty litter; place the mixture in a container; and then dispose it in the trash. The FDA also published a list of drugs recommended for disposal by flushing, [13].

Overall, the issue of PhACs in the environment is a recognized problem in the USA, and the recent implementation of the Secure and Responsible Drug Disposal Act allows more opportunities to safely collect the public's unused drugs. However, federal and state governments have been hesitant to adopt Extended Producer Responsibility-EPR approaches. EPR mean contribution or involvement of the producer (manufacturer) in the safe collection and disposal of unused and expired products.

The USA also has an environmental assessment (EA) process for drugs. The FDA is mandated under the National Environmental Policy Act (1970) to consider the environmental impacts of approving drugs in the application process (FDA, 1998), [14].

\section{The Canadian know-how}

All three levels of government in Canada have a role in waste management. The provincial/territorial governments predominately regulate waste management as they are responsible for issuing licenses and approvals, monitoring operations, and promoting waste diversion. Municipalities tend to be responsible for operating waste management services such as collection, transportation and disposal/recycling facilities. Under the Constitution Act, 1867, municipal institutions are an exclusive power of the provincial government. The provinces set out the powers delegated to municipalities by statute. The federal government also plays a role in waste issues relating to toxic substances, international movement of waste materials, federal lands and operations, and air emissions, [15].

With environment being a matter of shared jurisdiction, the Canadian Council of Ministers of the Environment (CCME) was formed for intergovernmental discussion of environmental issues. The CCME consists of environment ministers from the federal, provincial and territorial governments and serves "as a principal forum for members to develop national strategies, norms, and guidelines that each environment ministry across the country can use", [16]. In 1989, the CCME deemed waste management a problem and developed a national packaging policy to reduce packaging waste sent to landfills, [17]. They developed schedules and targets for waste minimization, including a $50 \%$ reduction in waste generation by 2000. In 2009, the CCME approved the Canada-wide Action Plan for EPR which outlined coordinated policies for creating EPR approaches, and set out timelines for implementing EPR laws for priority products. Drugs and sharps were identified as phase I priority which meant that EPR laws for these materials should be implemented by 2015. Thus, the CCME's strategies have the potential to harmonize provincial waste management frameworks. A Canada-wide medications 
return program would be challenging to adopt since waste management is mainly governed by the provinces. Hence, there are various provincial, municipal, and community initiatives that collect the public's unused drugs. Since waste policies tend to be promoted by the provinces, the discussion of post-consumer pharmaceutical waste strategies will begin with the provinces, followed by the municipalities and then the federal government. Currently, provincial governments play the greatest role in managing this waste.

The majority of provinces have some type of collection system for the public's post-consumer drugs. In general, Canadian consumers can return their unused drugs to pharmacies/ depots for safe collection, and incineration is the most common end-of-life treatment. Return programs tend to be initiated by governments, industry or pharmaceutical associations. Unused drugs from residential consumers are accepted while waste from industry (e.g. hospitals, dental offices or doctor offices) tends to be outside the scope of these programs.

\section{Discussions}

\section{Gulf region pharmaceutical waste (KSA)}

The extent of drugs wastage in the Gulf region, might constitute a significant figures compared to other part of the world. A study has been conducted by a Saudi clinical pharmacist with Doctorate credentials to shed some light on the extent of pharmaceutical wastage among families in the Arabian Gulf countries in general and in Kingdom Saudi Arabia-KSA in particular, [18]. In this study a questionnaire was administered in 1554 households in 5 regions in Saudi Arabia and 87 households in all other Gulf countries (Qatar, Kuwait, KSA, Sultanate Oman, UAE) except Bahrain. The study reached to a conclusion that, the total dollar value of medication waste in households was estimated to be in the close vicinity of 150 million dollars a year from the budget allocated to health care.

\section{UAE understandings}

In an article published in the United Arab Emirates Gulf News on October 2013 with the heading "Sick truth about how medicines go to waste in UAE",[19] the author stated that the gross abuse of insurance in the UAE is resulting in a shocking wastage of medicines worth millions of dirhams. A landmark study of 2.7 million claims conducted by Accumed PM, a Dubaibased healthcare consultancy at that time, has found that the average value of medicines in an insurance claim is as high as 23.31 per cent. Industry experts said the wastage occurs most commonly with painkillers, paracetamol, anti-histamines, cough syrups; nasal sprays etc., where the consumption is invariably less than what is sold by pharmacies. Even antibiotics end up in surplus, despite being prescribed in specific quantities for a specific duration. But pharmacies contend that barring over-thecounter drugs, they are required by law to sell medicines as entire units. Any attempt to do otherwise would amount to tampering, a legal violation.

\section{Sultanate of Oman familiarities}

Recently the same issue come to light in an article published in the Oman Daily Observer (issue of July 18, 2016), [20] with the heading "Medicines worth millions go waste" and the reasons is the same as that highlighted in the article of the UAE Gulf News.

\section{Kuwait}

A study conducted in Kuwait examined the practices, awareness and opinions of hospital pharmacists working in government hospitals regarding the disposal of unwanted pharmaceuticals. The findings would appear to support this call for further research. It was found that although the pharmacists were aware of the harmful impact of the improper disposal of unwanted pharmaceuticals, their disposal practices were suboptimal, [21].

\section{Management of pharmaceuticals waste}

The management of pharmaceutical waste is of paramount importance to avoid improper disposal with adverse outcomes on environment, land, public and depletion of resources.

Pharmaceuticals are ideally disposed by high temperature

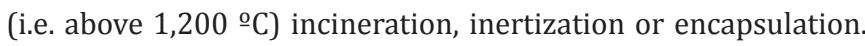

Table 1: The cost of pharmaceuticals waste

\begin{tabular}{|c|c|c|c|c|}
\hline Country & Cost of pharmaceuticals waste in USA Dollar & $\begin{array}{c}\text { Fiscal } \\
\text { year }\end{array}$ & Type of study & reference \\
\hline $\mathbf{K S A}^{* 15}$ & $28.8^{* *}$ & 2003 & Questionnaire & $\begin{array}{c}\text { Hisham et al } \\
{[18]}\end{array}$ \\
\hline $\mathbf{U S A}^{\dagger 15}$ & $\begin{array}{l}2.4 \text { to } 5.4 \\
\text { billions }\end{array}$ & 2014 & $\begin{array}{l}\text { Cross-sectional, observational two- } \\
\text { phased study }\end{array}$ & $\begin{array}{c}\text { Law et al } \\
\text { [23] }\end{array}$ \\
\hline Oman $^{15}$ & $37.2^{* *}$ & 2003 & Questionnaire & $\begin{array}{c}\text { Hisham et al } \\
{[18]}\end{array}$ \\
\hline $\mathbf{U K}^{\dagger+18}$ & $399.72^{* *}$ & 2015 & Case Study & $\begin{array}{c}\text { Bertie and } \\
\text { Ross et al } \\
\text { [22] }\end{array}$ \\
\hline $\mathbf{U A E} \S^{15}$ & $33.15^{* *}$ & 2003 & Questionnaire & $\begin{array}{c}\text { Hisham et al } \\
{[18]}\end{array}$ \\
\hline
\end{tabular}

Key: King Saudi Arabia $={ }^{*} \mathrm{KSA}$; ${ }^{* *}$ Millions; United States of America $=\mathrm{USA}^{\dagger}$; United Kingdom= UK ${ }^{\dagger+}$; United Arab Emirates= UA ${ }^{\mathrm{E}} \S$ 
Such incineration facilities, equipped with adequate emission control, are mainly to be found in the industrialized world.

Feasibility wise, the procedure of disposal, might constitute a burden for the management of pharmaceutical waste, as whose responsibility to cover such cost will be a question. However, at the same time presence of proper system for pharmaceutical waste collection, an area where pharmacists can play a major role will inform policy maker, healthcare providers and consumers about the issue, which may lead to an overall proper inventory control, good prescribing and dispensing practices, consumers commitment. Ultimately, this will lead to minimize the disposed pharmaceutical waste.

\section{Conclusion}

\section{Pharmacist's role in disposal of pharmaceuticals}

There is a dearth for conducting pharmaceuticals waste disposal strategies in health care facilities particularly in hospitals, long-stay and nursing homes. The clinical pharmacist is best placed healthcare professional to delve deeper, conduct, implement and monitor such strategies.

The most fundamental aspect role that can be performed by pharmacist is to minimize the amount of pharmaceutical waste and to ensure safe disposal of pharmaceutical waste.

Pharmacists can play a major role in this regard, by conducting scientific research and surveys, raise awareness among consumers about the importance of safe disposal of unused and expired pharmaceuticals and encourage policy makers to implement medication retain and disposal programs in accordance with existing guidelines and laws. Pharmacist counseling of patients at the dispensing time about safe disposal of pharmaceuticals is of paramount importance to strategic promotion and successful implementation of disposal programs.

Health authorities need to develop disposal programs as part of pharmaceuticals use analysis in health strategies and community initiatives to promote vigilant culture for undesirable environmental consequences in the developing and underdeveloped countries.

A unified disposal program should be in place where defined pharmaceuticals, prescription, non-prescription, type of formulation, narcotics and physician samples clearly been defined and addressed accordingly.

Finally, the key to solve the issue of pharmaceutical waste disposal is collaborative effort and co-operation between all the parties in the health care hierarchy. Established and applied laws, along with community awareness will definitely make our aim easily achievable.

\section{References}

1. Koshy S. Disposal of unwanted medications: throw, bury, burn or just ignore? Int J Pharm Pract. 2013;21(2):131-134. doi:10.1111/j.2042-7174.

2. Taylor D. Ecopharmacostewardship- A Pharmaceutical Industry Perspective. Green and Sustainable Pharmacy. 2010;105-126. doi.

\section{org/10.1007/978-3-642-05199-9_7}

3. The management of post-consumer pharmaceutical waste: A comparison between British Columbia and Ontario programs. Lisa Kingsmore, Master of Applied Science; Environmental Applied Science and Management; Ryerson University, 2013.

4. Benotti MJ, Trenholm RA, Vanderford BJ, Holady JC, Stanford BD, Snyder SA. Pharmaceuticals and endocrine disrupting compounds in U.S. drinking water. Environ Sci Technol. 2009;43(3):597-603.

5. Environment Canada. Pollution and waste - Managing solid waste. 2012.

6. World Health Organization-WHO. (1999). Guidelines for safe disposal of unwanted pharmaceuticals in and after emergencies. Available from http://www.who.int/ water_sanitation_health/medicalwaste/ unwantpharm.pdf.

7. World Health Organization. Pharmaceutical Products. Health Topics. 2013.

8. European Environment Agency. Pharmaceuticals in the environment Results of an EEA workshop. 2010.

9. Vollmer G. Disposal of pharmaceutical waste in households - A European survey. Green and sustainable pharmacy. 2010;165-178. doi.org/10.1007/978-3-642-05199-9_11

10. The National Return and Disposal of Unwanted Medicines Limited. Collections.

11. Brushin B. Return unwanted medicines survey- Victorian report. Melbourne Metropolitan Area. Melbourne, Australia. 2005.

12. United States Drug Enforcement Administration. National take-back initiative. 2013.

13. United States Food and Drug Administration. [U.S. FDA]. (2013a, Feb. 13). Drugs - Disposal of unused medicines: What you should know. Retrieved from http://www.fda.gov/drugs/resourcesforyou/ consumers/buyingusingmedicinesafely/ensuringsafeuseofmedicine/ safe disposalofmedicines/ucm186187.htm

14. United States Food and Drug Administration. Guidance for industry Environmental assessment of human drug and biologics applications. 1998.

15. Environment Canada. Pollution and waste - Managing solid waste. 2012.

16. Canadian Council of Ministers of the Environment. About CCME. 2011.

17. Canadian Council of Ministers of the Environment [CCME]. (1998, Jan.).National packaging protocol 1996 milestone report. Available from http://www.ccme.ca/assets/pdf/ milestone_e.pdf

18. Hisham S AbouAuda. An economic assessment of the extent of medication use and wastage among families in Saudi Arabia and Arabian Gulf countries. Clin Ther. 2003;25(4):1276-1292.

19. Sharmila Dhal. Chief Reporter; Gulf News, United Arab Emirates. 2013.

20. Samuel Kutty. Oman Daily Observer; Oman. 2016.

21. Abahassain E, Waheedi M, Koshy S. Practice, awareness and opinion of pharmacists toward disposal of unwanted medications in Kuwait. Saudi Pharm J. 2012; 20(3):195-201. doi:10.1016/j.jsps.2012.04.001

22. Bertie Hazell, Ross Robson. Pharmaceutical waste reduction in the NHS (UK National Health Service): A best practice compilation paper. 2015;1(6).

23. Law AV, Sakharkar P, Zargarzadeh A, Tai BW, Hess K, Hata M, et al. Taking stock of medication wastage: Unused medications in US households. Res Social Adm Pharm. 2015;11(4):571-578. doi: 10.1016/j.sapharm.2014.10.003 